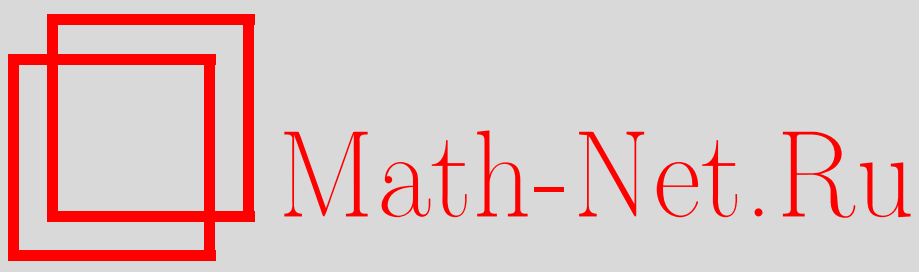

В. А. Васильев, О пространствах полиномиальных узлов, Матем. сб., 1996, том 187, номер 2, 37-58

DOI: https://doi.org/10.4213/sm108

Использование Общероссийского математического портала Math-Net.Ru подразумевает, что вы прочитали и согласны с пользовательским соглашением

http: //www.mathnet.ru/rus/agreement

Параметры загрузки:

IP: 54.147 .182 .235

26 апреля 2023 г., 13:46:47 
УДК 515.1

\section{В.А. Васильев}

\section{О пространствах полиномиальных узлов}

Вычисленыгрупшы гомологий неособых полиномиальных вложений $\mathbb{R}^{1} \rightarrow \mathbb{R}^{n}$ степени $\leqslant 4$. Развита общая алгебраическая техника подобных вычислений для пространств полиномиальных узлов произвольной степени.

Библиограбоя: 7 названий.

\section{§1. Введение}

Пространство узлов в $S^{n}$ с фиксированньм поведением вблизи отмеченной точки может быть аппроксимировано пространствами полиномиальных вложений $\Phi: \mathbb{R}^{1} \rightarrow \mathbb{R}^{n}$ вида

$$
\left\{\begin{array}{l}
x_{1}(t)=t^{d}+a_{1}^{1} t^{d-1}+\cdots+a_{d-1}^{1} t \\
\ldots \ldots \ldots \ldots \ldots \ldots \cdots \cdots \cdots \cdots \cdots \cdots \cdots \cdots \\
x_{n}(t)=t^{d}+a_{1}^{n} t^{d-1}+\cdots+a_{d-1}^{n} t
\end{array}\right.
$$

Например, любой узел класса $C^{1}$ в $S^{3}$ изотопически эквивалентен замыканию образа такого отображения с достаточно большим $d$. В [1] А. Шастри показал, что трилистниковые узлы могут быть реализованы такими вложениями с $d=5$. Мы доказываем, в частности, что никакой нетривиальный узел не реализуется отображениями степени меньшей чем 5 .

Обозначим через $\mathscr{K}_{d}^{n}$ пространство всех отображений $\Phi$ вида (1), а через $\Sigma \equiv \Sigma_{d}$ дискриминант $\mathscr{K}_{d}^{n}$, т.е. замыкание множества отображений (1), имеющих либо особые (с $\left.\Phi^{\prime}(t)=0\right)$, либо кратные точки.

Основной результат настоящей работы - следующая теорема.

ТЕОРема 1. Для любого $n \geqslant 2$ пространство $\mathscr{K}_{3}^{n} \backslash \Sigma$ стягиваемо, а пространство $\mathscr{K}_{4}^{n} \backslash \Sigma$ гомологически эквивалентно $S^{n-2}$. При $n \geqslant 4$ пространство $\mathscr{K}_{4}^{n} \backslash \Sigma$ также и гомотопически эквивалентно $S^{n-2}$. Образующая группьи $H^{n-2}\left(\mathscr{K}_{4}^{n} \backslash \Sigma\right)$ равна индексу зацепления в $\mathscr{K}_{4}^{n}$ с множсеством отображений (1), имеющих особие точки.

Первое утверждение теоремы тривиально и, вероятно, известно специалистам. Действительно, легко проверить что пространство $\mathscr{K}_{3}^{n} \backslash \Sigma$ звездно относительно своей точки (1) с $x_{1}(t) \equiv \cdots \equiv x_{n}(t) \equiv t^{3}+t$.

Работа вьполнена при поддержке Российского фонда фундаменталњных исследований (грант № 95-01-00846а) и Международного научного фонда (грант № MQO000/MQO300).

$$
\text { (C) В.А. ВАСильев } 1996
$$


ПРЕДЛОЖЕНИЕ 1. Если $d$ нечетно, то пространство отображений (1) $c$ кратными или особыми точками замкнуто и, следовательно, совпадает с $\Sigma$. Если d четно, то $\Sigma$ есть обвединение такого пространства и пространства всех отображений (1) с $a_{1}^{1}=\cdots=a_{1}^{n}$.

ДокАЗАтЕльство. Для любого $\alpha \in \mathbb{R}$ пространство отображений (1) с $a_{1}^{1}=$ $\cdots=a_{1}^{n}=\alpha$-это предельное положение пространств отображений (1), склеивающих точки $t$ и $\alpha-t \in \mathbb{R}^{1}, t \rightarrow \infty$.

ПРЕДЛОЖЕНИЕ 2. Если $n \geqslant 5$ или $n=d=4$, то пространство $\mathscr{K}_{d}^{n} \backslash \Sigma$ односвязно. (В частности, гомотопическая часть теоремы 1 вытекает из гомологической в силу теоремь Уайтхеда.)

ДокАЗАТЕльство. $\Sigma$ есть полуалгебраическое множество коразмерности $n-2$, поэтому наше предложение верно при $n \geqslant 5$, а в случае $n=4$ группа $\pi_{1}\left(\mathscr{K}_{d}^{n} \backslash \Sigma\right)$ порождена несколькими простыми петлями, охватьвающими множество $\Sigma$ вблизи его неособых точек. Более того, как мы увидим в $\S 4$, если $d \geqslant 4$, то $\Sigma$ обладает разрешением особенностей $F_{1} \sigma \rightarrow \Sigma$, где $F_{1} \sigma$ - связное гладкое многообразие с непустой границей, и коразмерность в нем полного прообраза множества особых точек $\Sigma$ равна $n-2$. Следовательно, любая простая петля в $\mathscr{K}_{4}^{n} \backslash \Sigma$, охватывающая $\Sigma$ вблизи неособой точки $\xi \in \Sigma$, может быть отцеплена от $\Sigma$ при помощи деформации над путем, соединяющим $\xi$ с $\partial \Sigma$ в неособой части $\Sigma$.

ПРЕДЛОЖЕНИЕ 3. Для любого четного $d$

а) пространство $\mathscr{K}_{d}^{3} \backslash \Sigma$ является произведением некоторого топологического пространства на окружность;

b) для любого $n \geqslant 3$, в группе $H^{n-2}\left(\mathscr{K}_{d}^{n} \backslash \Sigma\right)$ имеется по меньшей мере одна свободная образующая: индекс зацепления с пространством отображсений (1) с $a_{1}^{1}=\cdots=a_{1}^{n}$;

c) этот индекс равен индексу зацепления $с$ (подходящим образом ориентированным) мнохсеством отображсний, имеющих особъе точки.

ДокАЗАТЕльство. Группа $S^{1}$ вращений $\mathbb{R}^{3}$ вокруг оси $(1,1,1)$ свободно действует на пространстве отображений (1), коэффициенты $a_{1}^{1}, a_{1}^{2}, a_{1}^{3}$ которых не все одинаковы, и любая орбита этого действия содержит единственное отображение (1) с $a_{1}^{1}=a_{1}^{2}$ и $a_{1}^{1}+a_{1}^{2}-2 a_{1}^{3}>0$. Это доказывает утверждение а). Индексы зацепления всех этих орбит с множеством отображений с $a_{1}^{1}=a_{1}^{2}=a_{1}^{3}$ равны 1 . (При $d=4$ тождественное вложение любой из этих орбит в $\mathscr{K}_{d}^{3} \backslash \Sigma$ индуцирует гомологическую эквивалентность, обещанную в теореме 1.)

Для произвольного $n$ индекс зацепления, указанный в утверждении b), нетривиален, поскольку орбита отображения (1) с $x_{1}(t) \equiv \cdots \equiv x_{n-1}(t) \equiv t^{d}, x_{n}(t) \equiv t^{d}+$ $t^{d-1}+t$, при аналогичном действии групшы $S O(n-1)$ вращений, сохраняющих ось $(1, \ldots, 1)$, диффеоморфна $S^{n-2}$, и наш индекс зацепления равен на ней \pm 1 . Утверждение с) будет доказано в п. 4.1.

Наш метод вычисления гомологий в принципе может быть применен к когомологиям пространств полиномиальных узлов произвольной степени $d$. При $n=3$ и $d \rightarrow \infty$ эти когомологии стабилизируются к группам когомологий пространства узлов, включающим пространство всех инвариантов узлов. Коциклы (в частности инварианты), которые стабилизируются хорошо, были введены в [2], [3]. Здесь 
мы начинаем регулярное исследование произвольных (нестабильных) коциклов и процесса их стабилизации.

\section{§2. Конические разрешения дискриминанта}

Как и в предыдуших работах [2], [3], мы используем идею из [4]: мы рассматриваем группу замкнутых гомологий дискриминанта

$$
\bar{H}_{*}(\Sigma)
$$

т.е. группу гомологий одноточечной компактификации $\Sigma$, приведенную по модулю добавленной точки. Группа когомологий $\mathscr{K}_{d}^{n} \backslash \Sigma$ двойственна по Александеру этой группе.

Для вычисления группы (2) мы строим конические разрешения дискриминантных многообразий (ранее использовавшшеся в [5]), которые являются непрерывньм аналогом симплициальных разрешений, активно используемых в алгебраической геометрии, см. также [3], [6].

2.1. Первичное коническое разрешение. В нашей ситуации простейшие конические разрешения строятся следуюшим образом. Обозначим через $P_{d}$ пространство полиномов вида

$$
t^{d}+a_{1} t^{d-1}+\cdots+a_{d-1} t
$$

так что $\mathscr{K}_{d}^{n} \simeq\left(P_{d}\right)^{n}$. Рассмотрим несвязное объединение всех грассмановых многообразий $G_{i}\left(P_{d}\right) i$-мерных аффинных подпространств в $P_{d}, i=0, \ldots, d-2$. Искомое коническое разрешение является подмножеством прямого произведения пространства $\mathscr{K}_{d}^{n}$ и джойна этих грассманианов

$$
G_{0}\left(P_{d}\right) * G_{1}\left(P_{d}\right) * \cdots * G_{d-2}\left(P_{d}\right) .
$$

ОПРЕДЕЛЕНИЕ 1. Элементарное условие на полиномы $f \in P_{d}$ - это условие одного из следуюших трех типов:

a) для некоторой отмеченной пары точек $t \neq s \in \mathbb{R}^{1}, f(t)=f(s)$;

b) для некоторой отмеченной точки $t, f^{\prime}(t)=0$;

c) (только если $d$ четно) коэффициент $a_{1}$ полинома $f$ равен некоторому отмеченному числу $\alpha$.

Пространство всех элементарных условий обладает естественной топологией: при нечетных $d$, оно гомеоморфно замкнутой полуплоскости

$$
\left\{(t, s) \in \mathbb{R}^{2} \mid t \leqslant s\right\},
$$

где диагональ $\{t=s\}$ состоит из условий типа b). Для четных $d$ это пространство гомеоморфно бесконечной полосе $\mathbb{R}^{1} \times[0,1]$ и получается из описанной выше полуплоскости добавлением прямой "на бесконечности": точка $\{\alpha\}, \alpha \in \mathbb{R}$, этой прямой есть обшая предельная точка всех некомпактных кривых в (5), стремящихся к бесконечности таким образом, что значения функции $t+s$ на них стремятся 


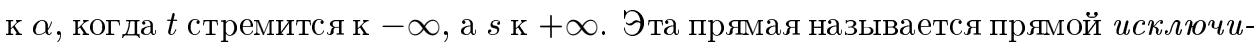
тельных условий.

В обоих случаях мы обозначаем это пространство через $\Psi(d)$.

Если $d>2$, то любое элементарное условие определяет точку многообразия $G_{d-2}\left(P_{d}\right)$, а именно пространство всех полиномов, удовлетворяющих этому условию. Любая точка в $G_{d-2}\left(P_{d}\right)$ соответствует не более чем одному элементарному условию; описанная выше топология в пространстве $\Psi(d)$ - это просто топология, индуцированная при этом соответствии из стандартной топологии в $G_{d-2}\left(P_{d}\right)$.

Любой набор $T$ элементарных условий определяет аффинное подпространство $L(T)$ в $P_{d}$, состоящее из полиномов, удовлетворяющих всем этим условиям, а, следовательно, точку в некотором $G_{i}\left(P_{d}\right)$.

Обозначим через $C(d, i)$ множество всех $i$-мерных аффинных подпространств вида $L(T)$ в $P_{d}$, а через $\bar{C}(d, i)$ его замыкание в $G_{i}\left(P_{d}\right)$.

Джойн (4) это, грубо говоря, снабженное естественной топологией объединение всех симплексов, вершины которых принадлежат множествам $G_{i}\left(P_{d}\right)$ с различными $i$.

ОПРЕДЕЛЕНИЕ 2. Симплекс (произвольной размерности) этого джойна называется когерентным, если все его вершины суть точки подмножеств $\bar{C}(d, i) \subset G_{i}\left(P_{d}\right)$ с различными $i$, и все плоскости в $P_{d}$, соответствующие этим вершинам, инцидентны друг другу.

Обозначим через $\Lambda(d)$ объединение всех непустых когерентных симплексов в джойне (4).

Любому из симплексов $\Delta$, составляющих $\Lambda(d)$, соответствует аффинная плоскость $L(\Delta) \subset P_{d}$ (а именно та из вершин симплекса $\Delta$, которая лежит в $G_{i}\left(P_{d}\right)$ с наименьшим значением $i)$, а, следовательно, также и некоторая плоскость $\mathscr{L}(\Delta)$ в $\mathscr{K}_{d}^{n}$ : множество отображений вида (1), все $n$ компонент которых принадлежат $L(\Delta)$.

Коническое разрешение дискриминанта, $R \Sigma$, определяется как подмножество в $\Lambda(d) \times \mathscr{K}_{d}^{n}$, состояшее из пар вида (внутренняя точка некоторого когерентного симплекса $\Delta$, точка соответствуюшей ей плоскости $\left.\mathscr{L}(\Delta) \subset \mathscr{K}_{d}^{n}\right)$.

Определим отображение $\pi: R \Sigma \rightarrow \Sigma$ как ограничение на $R \Sigma$ очевидной проекции $\Lambda(d) \times \mathscr{K}_{d}^{n} \rightarrow \mathscr{K}_{d}^{n}$.

ПРЕДЛОЖЕНИЕ 4. Для любого $d$ отображсение $\pi$ собственно и сюрвективно. Оно определяет гомотопическую әквивалентность одноточечных компактификащий пространств $R \Sigma$ u $\Sigma$. $R \Sigma$ гомеоморфно полуалгебраическому множеству таким образом, что $\pi$ становится кусочно-алгебраическим отобрахсением.

ДокАЗАтЕльство. Первое утверждение очевидно, а последнее вытекает из построения, если мы реализуем джойн (4) как объединение симплексов, натянутых на точки грассманианов $G_{i}\left(P_{d}\right)$, вложенных алгебраически и в общем положении в евклидово пространство очень большой размерности. Второе утверждение вытекает теперь из того, что прообраз любой точки $\varphi \in \Sigma$ есть стягиваемый компактный $C W$-комплекс, а именно, объединение всех когерентных симплексов $\Delta$, обшая главная вершина $L(\Delta)$ которых есть минимальная аффиннная плоскость в $P_{d}$, 
принадлежашая некоторому $\bar{C}(d, i)$ и содержашая одновременно все $n$ компонент отображения $\varphi$.

2.2. Приведенное коническое разрешение. Как правило, построенное выше разрешение не является самьм удобньм и его можно несколько упростить ради сокрашения вычислений.

ПримеР. Типичная плоскость $L(T)$ коразмерности 2 в $P_{d}, d \geqslant 4$, выделяется двумя различными элементарньми условиями $x, y \in \Psi(d)$ типа а) из определения 1. Такая точка $L(T) \in G_{d-3}\left(P_{d}\right)$ соединена когерентными отрезками с ровно двумя плоскостями $L(x), L(y) \in G_{d-2}\left(P_{d}\right)$. Однако при $x$ стремящемся к $y$ предельное значение $L(x, y)$ соединено с единственной точкой $L(y) \in G_{d-2}\left(P_{d}\right)$; это предельное значение принадлежит $\bar{C}(d, d-3)$, но не $C(d, d-3)$. (Такие предельные значения при фиксированном $y$ пробегают окружность и зависят от угла атаки $x$ на $y$; эти предельные плоскости в $P_{d}$ заметают плоскость $L(y)$ и определяют $\sigma$-процесс в ней с центром в пространстве полиномов с нулевьми производными в обеих точках, составляющих пару $y$.)

В общем случае, для любой плоскости $L \in \bar{C}(d, i)$ обозначим через $\bar{L}$ минимальную плоскость вида $L(T)$, содержашую $L$ : по определению, это точка множества $C(d, j)$ с некоторым $j \geqslant i$. Обратно, любой точке $L^{\prime} \in C(d, j)$ соответствует подкомплекс в $\Lambda(d)$, состоящий из всех точек $L \in \bar{C}(d, i), i \leqslant j$, таких что $L^{\prime}=\bar{L}$, и из всех когерентных симплексов, натянутых на такие точки с разными $i$. Очевидно, что этот подкомплекс компактен и стягиваем.

Редущирующее отображсене $\mathrm{red}: \Lambda(d) \rightarrow \widetilde{\Lambda}(d)$ определяется следующим образом: для любого $i$ и любого $L \in \bar{C}(d, i)$ мы отображаем точку $L$ в $\bar{L}$ и продолжаем это отображение по линейности на все когерентные симплексы; образ когерентного симплекса под действием этого отображения, очевидно, вновь когерентен. Затем мы отождествляем между собой все точки в $\Lambda(d)$, отображаемые этим продолженным отображением в одну и ту же точку. Пространство $\widetilde{\Lambda}(d)$ определяется как факторпространство $\Lambda(d)$ относительно этого отождествления.

Это отображение очевидным образом определяет отображение $\Lambda(d) \times \mathscr{K}_{d}^{n} \rightarrow$ $\widetilde{\Lambda}(d) \times \mathscr{K}_{d}^{n}$, а, следовательно, и отображение разрешенного дискриминанта $R \Sigma$ на некоторьй подкомплекс $\widetilde{R} \Sigma \subset \widetilde{\Lambda}(d) \times \mathscr{K}_{d}^{n}$.

ПРЕДЛОЖЕНИЕ 5. Продолженное отображение отождествления

$$
\operatorname{Red}: R \Sigma \rightarrow \widetilde{R} \Sigma
$$

задает гомотопическую әквивалентность одноточечных компактификаций пространств $R \Sigma$ и $\widetilde{R} \Sigma$ и факторизует отображсение $\pi: R \Sigma \rightarrow \Sigma$ (т.е. существует собственное отображсение $\widetilde{\pi}: \widetilde{R} \Sigma \rightarrow \Sigma$ такое, что $\pi=\widetilde{\pi} \circ \mathrm{Red})$.

Доказательство следует непосредственно из построения.

2.3. Фильтрации и спектральные последовательности. Пространство $\Lambda(d)$ обладает естественной возрастающей фильтрацией. Действительно, фильтрация любой внутренней точки симплекса $\Delta \subset \Lambda(d)$ равна коразмерности $d-1-i$ главной плоскости $L(\Delta) \in \bar{C}(d, i)$ этого симплекса. Следовательно, индуцируется также фильтрация на пространстве $\widetilde{\Lambda}(d)$ : фильтрация класса эквивалентности 
$x \in \widetilde{\Lambda}(d)$ равняется наименьшему значению фильтраций точек в $\Lambda(d)$, составляюших $x$. Очевидные проекции $R \Sigma \rightarrow \Lambda(d), \widetilde{R} \Sigma \rightarrow \widetilde{\Lambda}(d)$ индуцируют фильтрации также и на пространствах $R \Sigma$ и $\widetilde{R} \Sigma$. По построению, член $F_{i} \backslash F_{i-1}$ этой фильтрации на $R \Sigma$ (соответственно, на $\widetilde{R} \Sigma)$ является пространством $n(d-1-i)$-мерного аф̆финного расслоения над аналогичньт членом фильтрации на $\Lambda(d)$ (соответственно, на $\widetilde{\Lambda}(d))$.

Эта фильтрация определяет спектральную последовательность, сходящуюся к группе (2): ее член $E_{p, q}^{1}$ изоморфен $\bar{H}_{p+q}\left(F_{p} \backslash F_{p-1}\right)$. По ряду причин (см., например, [3]) удобно переименовать этот член в $E_{1}^{-p, n(d-1)-1-q}$ так, что спектральная последовательность становится когомологической; эта перевернутая спектральная последовательность сходится в точности к когомологиям пространства $\mathscr{K}_{d}^{n} \backslash \Sigma$.

\section{§3. Тривиальный пример}

Хотя топология пространства $\mathscr{K}_{3}^{n} \backslash \Sigma$ ясна, сейчас мы вычислим его когомологии, чтобы продемонстрировать наш общий метод.

ОПРЕДЕЛЕНИЕ 3 . Открытый конус над топологическим пространством $X$ это обычный конус $C X$ с выброшенной базой $X$.

Член $F_{1}$ естественной фильтрации на $\widetilde{\Lambda}(3)$ (и на $\Lambda(3)$ ) - это пространство $C(3,1) \equiv \Psi(3)$, а аналогичньй член фильтрации на $\widetilde{R} \Sigma$ является пространством $n$-мерного аффинного расслоения над $\Psi(3)$. (Именно, любой элемент $x \in \Psi(3)$ определяет (одномерное) семейство полиномов в $P_{3}$; слой над $x$ обещанного расслоения является прямой суммой $n$ копий этого семейства.) В частности, $E_{1}^{-1, *} \equiv 0$.

Подпространства $L(T)$ коразмерности 2 в $P_{3}$ - это просто точки, поэтому для того, чтобы вычислить член $E_{1}^{-2, *}$, мы рассмотрим все полиномы (3) степени $d=3$ и для любого такого полинома $f$ возьмем конус, база которого есть пространство всех аффинных прямых $L(x) \in C(3,1) \equiv \Psi(3)$, содержаших $f$; член $F_{2} \backslash F_{1}$ пространства $R \Sigma$ заметается всеми такими (открытыми) конусами (основания их лежат в члене $\left.F_{1}\right)$. Для вычисления его гомологий нам потребуется следующее понятие.

ОПРЕДЕЛЕНИЕ 4. Кривая отношений $r(f)$, определяемая полиномом $f: \mathbb{R}^{1} \rightarrow \mathbb{R}^{1}$, - это множество всех пар $(t, s) \in \Psi(d)$ таких, что $f(t)=f(s)$ (если $t \neq s$ ) или $f^{\prime}(t)=0$ (если $t=s$ ); если $d$ четно, то эта кривая содержит дополнительно точку исключительной прямой в $\Psi(d)$, равную коэффициенту $a_{1}$ полинома $f$.

ЛЕмма 1. Если $f \in P_{d}, d$ нечетно, то кривая $r(f)$ лежит в "конечной" области в $\Psi(d)$. Если d четно, то ровно одна ветвь $r(f)$ уходит на бесконечность, и прямая $\left\{t+s=a_{1}\right\}$ является ее асимптотой.

Полиномы $f_{1}, \ldots, f_{k}$ принадлежат подпространству $L(T)$ (см. п. 2.1) тогда и только тогда, когда множество $r\left(f_{1}\right) \cap \cdots \cap r\left(f_{k}\right)$ содержит $T$.

Кривая отношений кубического полинома $f=t^{3}+\alpha t^{2}+\beta t$ задается уравнением $\left(t^{2}+t s+s^{2}\right)+\alpha(t+s)+\beta=0$, следовательно, либо пуста (если $f^{\prime}$ всюду положительно), либо состоит из единственной точки (если $f^{\prime}$ имеет двойной корень), либо является половиной эллипса, ортогональной к границе полуплоскости $\Psi(3)$ 
в своих двух концевых точках (которые суть такие диагональные точки $\{t, t\}$, что $t$ является корнем $\left.f^{\prime}\right)$.

Полиномы с $f^{\prime}>0$ (множество их выделяется в $P_{3}$ неравенством $\alpha^{2}<3 \beta$ ), очевидно, не принадлежат ни одному семейству $L(x)$ и соответствующий конус в $F_{2}$ пуст.

Пусть $f$ - произвольный полином с двумя критическими точками. Тогда подмножество в $F_{2} \backslash F_{1}$, соответствуюшее $f$, есть открытый конус над полуэллипсом ( отрезком) $r(f)$ : база этого конуса принадлежит $F_{1}$. Следовательно, часть $F_{2} \backslash F_{1}$, заметенная конусами, соответствуюшими таким $f$, является расслоением над 2-клеткой $\left\{(\alpha, \beta) \in \mathbb{R}^{2} \mid \alpha^{2}>3 \beta\right\}$ со слоем, гомеоморфньм треугольнику, из которого выброшена одна замкнутая сторона. (Замкнутые) гомологии этой части тривиальны.

Наконец, для граничных значений $f$ (т.е. $\alpha^{2}=3 \beta$ ) сегмент $r(f)$ в основании таких открытых конусов стягивается в точку, а, следовательно, конус, соответствующий такому $f$ в члене $F_{2} \backslash F_{1}$ естественной фильтрации $R \Sigma$, есть полуинтервал; при переходе к $\widetilde{R} \Sigma$ эти интервалы исчезают и дают тривиальный вклад в гомологии дискриминанта. Итак, столбец $E_{1}^{-2, *}$ также исчезает и вся группа $\bar{H}_{*}(\Sigma)$ тривиальна.

\section{§4. Пространство узлов четвертой степени}

4.1. Столбец $E_{1}^{-1, *}$. Для любого $d$ член $F_{1}$ стандартной фильтрации $\widetilde{R} \Sigma$ является пространством $n(d-2)$-мерного аффинного расслоения над $\Psi(d)$, следовательно, при четных $d$ группа $E_{1}^{-1, q}$ свободная циклическая, если $q=n-1$, и тривиальна для всех других $q$. Образуюшая группы $E_{1}^{-1, n-1}$ может быть реализована как фундаментальный класс (нормализованного) множества отображений (1) с особьми точками или (гомологичного ему) множества отображений с $a_{1}^{1}=\cdots=a_{1}^{n}$.

Это дает также доказательство утверждения с) предложения 3.

4.2. Столбец $E_{1}^{-2, *}$. Основной результат этого пункта - следующая теорема.

TEOPEMa 2. $E_{1}^{-2, *} \equiv 0$.

В соответствии с общим алгоритмом, мы должны рассмотреть все аффинные прямые в $P_{4}$ и для каждой из них взять открытый конус над множеством всех элементарных условий $x \in \Psi(4)$, которым удовлетворяют все полиномы этой прямой. Член $F_{2} \backslash F_{1}$ пространства $R \Sigma$ является пространством $n$-мерного аффинного расслоения над объединением всех таких конусов.

ЛЕмма 2. Число әлементарных условий, удовлетворяемых всеми полиномами из некоторой аффинной прямой в $P_{d}$, может равняться $0,1, \ldots$, $(d-1)(d-2) / 2$ или $\infty$.

ДокАЗАТЕЛЬСтво. Зафиксируем каноническое представление этих прямых. Прямая задается выбором любой ее точки $f$ и любого направляюшего вектора $g$, т.е. разности любых двух различных точек. Это направление $g$ является полиномом, степень которого строго меньше $d$. 
ОПРЕДЕЛЕНИЕ 5. Пара полиномов $(f, g)$, представляюшая прямую линию в $P_{d}$, является канонической, если старший коэффициент в $g$ равен 1 , а коэффициент при мономе $t^{\operatorname{deg} g}$ в $f$ равен 0 .

Очевидно, любая аффинная прямая в $P_{d}$ единственньм образом задается канонической парой полиномов $(f, g)$. Множество элементарных условий, удовлетворяемых всеми полиномами такой прямой, есть не что иное, как множество $r(f) \cap$ $r(g)$.

Кривая отношений полинома степени $d$ есть плоская кривая степени $d-1$, поэтому лемма 2 вытекает из теоремы Безу и из того, что кривые отношений инвариантны относительно отражения $(t, s) \rightarrow(s, t)$ и нас интересуют только точки с $t \leqslant s$.

В частности, при $d=4$ мощность множества $r(f) \cap r(g)$ может равняться $0,1,2,3$ или $\infty$. Очевидно, если это число бесконечно, то кривая $r(f)$ приводима.

ЛЕмма 3. Для полинома $f$ степени 4 кривая $r(f)$ приводима только если $f$ симметричен относительно некоторой точки $t_{0} \in \mathbb{R}^{1}$, m.е. $f=\left(t-t_{0}\right)^{4}+$ $\lambda\left(t-t_{0}\right)^{2}+c$ с некоторым $\lambda u c=-\lambda t_{0}^{2} . \quad B$ этом случае $r(t)$ состоит из отрезка $\left\{(t, s) \mid t+s=2 t_{0}\right\}$ и половины круга (возможно мнимого) $\left(t-t_{0}\right)^{2}+$ $\left(s-t_{0}\right)^{2}+\lambda=0$.

Очевидно, такой полукруг не может совпадать с кривой отношений полинома степени $\leqslant 3$, следовательно, пара $(f, g)$ удовлетворяет бесконечному числу элементарных условий, только если $\operatorname{deg} g=2$ и центры симметрии $g$ и $f$ в $\mathbb{R}^{1}$ совпадают.

Если прямая $(f, g) \subset P_{4}$ удовлетворяет 1 элементарному условию или не удовлетворяет ни одному, то над ней нет точек в члене $F_{2} \backslash F_{1}$ множества $\widetilde{R} \Sigma$ (во втором случае даже и в аналогичном члене множества $R \Sigma$ ); если она удовлетворяет 2 (соответственно, 3$)$ условиям, то соответствующее множество в $F_{2} \backslash F_{1}$ - открытый интервал (соответственно, звезда из трех лучей без концов).

4.2.1. Подправленное коническое разрешение. Сейчас мы слегка подправим конструкцию конического разрешения множества $\Sigma \subset P_{4}$, заменяя его (и каждый член $F_{i} \backslash F_{i-1}$ его естественной фильтрации) гомологически эквивалентньм пространством. Например, трехконечные звезды без конечных точек, соответствуюшие в канонической конструкции пространства $\Lambda(4)$ любой прямой в $P_{4}$, удовлетворяющей ровно трем условиям, будут заменены треугольниками без вершин.

ЛЕмма 4. Для любой пары различных әлементарных условий в $\Psi(4)$, не более одного из которых является исключительным, множество полиномов в $P_{4}$, удовлетворяющих әтим двум условиям, является аффинной прямой. Все аффинные прямые в $P_{4}$, точки которых удовлетворяют одновременно не менее, чем двум әлементарным условиям, могут быть заданы таким образом.

Обозначим через $\Psi^{[2]}(4)$ пространство неупорядоченных пар различных элементарных условий, по крайней мере одно из которых - неисключительное. Тогда мы получаем непрерывное отображение

$$
\nu: \Psi^{[2]}(4) \rightarrow \bar{C}(4,1)
$$


отображающее пару условий $(x, y)$ в семейство всех удовлетворяющих им полиномов. Пусть $\bar{\Psi}^{[2]}(4)$ - пополнение $\Psi^{[2]}(4)$, индуцированное этим отображением из пополнения $\bar{C}(4,1)$ пространства $C(4,1)$ (т.е. $\bar{\Psi}^{[2]}(4)$ есть объединение $\Psi^{[2]}(4)$ и некоторого множества $\partial \Psi^{[2]}(4)$, канонически гомеоморфиного $\bar{C}(4,1) \backslash C(4,1)$, топологизированное таким образом, что последовательность $\left\{x_{i}\right\} \in \Psi^{[2]}(4)$ сходится к точке $y \in \partial \Psi^{[2]}(4)$ тогда и только тогда, когда последовательность $\left\{\nu\left(x_{i}\right)\right\} \in C(4,1)$ сходится к точке множества $\bar{C}(4,1) \backslash C(4,1)$, соответствуюшей $y$ в силу канонического гомеоморфизма). Очевидное продолженное отображение $\bar{\Psi}^{[2]}(4) \rightarrow \bar{C}(4,1)$ вновь будет обозначено через $\nu$. Теперь рассмотрим джойн

$$
\bar{C}(4,0) * \bar{C}(4,1) * \bar{\Psi}^{[2]}(4) * \bar{C}(4,2)
$$

Любой точке множества $\bar{C}(4,0), \bar{C}(4,1), \bar{\Psi}^{[2]}(4)$ или $\bar{C}(4,2)$ соответствует аффинное подпространство в $P_{4}$ : для пространств $\bar{C}(4, i)$ это соответствие тавтологично, а точке $x \in \bar{\Psi}^{[2]}(4)$ соответствует прямая $\nu(x)$. Симплекс произвольной размерности в джойне (8) называется когерентным, если все плоскости в $P_{4}$, соответствуюшие его вершинам, инцидентны друг другу (в частности, если среди вершин этого симплекса есть точки как $\bar{C}(4,1)$, так и $\bar{\Psi}^{[2]}(4)$, то соответствующие прямые должны совпадать).

Последуюшее построение конических разрешений $\Sigma$, основанное на объединении когерентных симплексов в (8), повторяет конструкции из п.п. $2.1,2.2$; пространства этих разрешений обозначаются, соответственно, через $\rho \Sigma$ и $\widetilde{\rho} \Sigma$, объединение когерентных симплексов через $\lambda(4)$, а его естественное факторпространство (ср. п. 2.2) через $\widetilde{\lambda}(4)$.

Джойн (8) (а следовательно и его подпространство $\lambda(4))$ обладает естественной возрастающей фильтрацией: $F_{1}$ состоит из точек множества $C(4,2), F_{1.5}$ состоит из точек множеств $C(4,2)$ и $\bar{\Psi}^{[2]}(4)$ и соединяющих их отрезков; $F_{2}$ состоит из точек $\bar{C}(4,1), \bar{\Psi}^{[2]}(4)$ и $C(4,2)$ и соединяющих их отрезков и треугольников; наконец, $F_{3}$ это весь джойн $(8)$. Как и в п. 2.3, эта фильтрация порождает фильтрации на $\widetilde{\lambda}(4), \rho \Sigma$ и $\widetilde{\rho} \Sigma$.

Имеются естественные сохраняющие фильтрацию проекции

$$
\lambda(4) \rightarrow \Lambda(4), \quad \widetilde{\lambda}(4) \rightarrow \widetilde{\Lambda}(4)
$$

Например, первая из них линейно отображает любой когерентный симплекс из (8) на его грань, прина длежащую $\bar{C}(4,0) * \bar{C}(4,1) * \bar{C}(4,2)$, тождественна на этой грани и отображает вершину $x \in \bar{\Psi}^{[2]}(4)$ в вершину $\nu(x) \in \bar{C}(4,1)$. Вторая проекция в $(9)$ индуцирована первой. Эти проекции определяют также отображения

$$
\rho \Sigma \rightarrow R \Sigma, \quad \widetilde{\rho} \Sigma \rightarrow \widetilde{R} \Sigma
$$

действительно, отображения (9) очевидным образом поднимаются до отображений $\lambda(4) \times \mathscr{K}_{4}^{n} \rightarrow \Lambda(4) \times \mathscr{K}_{4}^{n}, \widetilde{\lambda}(4) \times \mathscr{K}_{4}^{n} \rightarrow \widetilde{\Lambda}(4) \times \mathscr{K}_{4}^{n} ;$ искомые отображения (10) - это их ограничения на подмножества $\rho \Sigma \subset \lambda(4) \times \mathscr{K}_{4}^{n}, \widetilde{\rho} \Sigma \subset \widetilde{\lambda}(4) \times \mathscr{K}_{4}^{n}$. 
ПРЕДЛОЖЕНИЕ 6. Проекиии (10) определяют гомотопичские әквивалентности всех участвующих в них пространств. Более того, для любого $i=$ $1,2,3$ то же верно для индуцированных ими отображений членов $F_{i} / F_{i-1}$ наших фильтраций пространств $\rho \Sigma$ (соответственно, $\widetilde{\rho} \Sigma$ ) на аналогичные члены $R \Sigma$ (соответственно, $\widetilde{R} \Sigma)$. В частности, эти отображсения индуцируют изоморфизмы соответствующих спектральных последовательностей.

(Добавленньй член $F_{1.5}$ фильтраций $\rho \Sigma$ и $\widetilde{\rho} \Sigma$ является просто вспомогательной конструкцией для вычисления груп $E_{1}^{-2, i}$ этих пространств.) Теперь теорема 2 вытекает из следующих двух лемм (описывающих введенные выше фильтрации пространства $\widetilde{\rho} \Sigma)$.

Лемма 5. $\bar{H}_{*}\left(F_{1.5} \backslash F_{1}\right) \equiv 0$.

Лemma $6 . \bar{H}_{*}\left(F_{2} \backslash F_{1.5}\right) \equiv 0$.

ДокАЗАТЕльство леммы 5 . Член $F_{1.5} \backslash F_{1}$ естественной фильтрации в $\tilde{\lambda}(4)$ гомеоморфен пространству локально тривиального расслоения над $\Psi^{[2]}$ (4) со слоем интервал: действительно, любой точке $\{x, y\} \in \Psi^{[2]}(4)$ соответствует объединение двух полуинтервалов $[\{x, y\} ; x)$ и $[\{x, y\} ; y)$. Легко вычисляется, что единственный нетривиальный элемент фундаментальной группы $\Psi^{[2]}(4)$ изменяет ориентации этого расслоения интервалов и каждого из $n$ линейных слагаемых канонического $n$-мерного аффинного расслоения $\left(F_{1.5} \backslash F_{1}\right)(\widetilde{\rho} \Sigma) \rightarrow\left(F_{1.5} \backslash F_{1}\right)(\widetilde{\lambda}(4))$. Следовательно, группа $\bar{H}_{i}\left(\left(F_{1.5} \backslash F_{1}\right)(\widetilde{\rho} \Sigma)\right)$ изоморфна группе $\bar{H}_{i-n-1}\left(\Psi^{[2]}(4)\right)$, если $n$ нечетно, и группе $\bar{H}_{i-n-1}\left(\Psi^{[2]}(4) ; \pm \mathbb{Z}\right)$ (где $\pm \mathbb{Z}-$ единственная нетривиальная локальная система со слоем $\mathbb{Z}$ ), если $n$ четно. Обе эти групшы легко вычисляются и оказываются тривиальньми.

4.3. Доказательство леммы 6. Член $F_{2} \backslash F_{1.5}$ пространства $\tilde{\lambda}(4)$ состоит из двух частей “ 3 ” и “ $\infty$ ”, заметаемых когерентными треугольниками

$$
\left[(f, g) \in G_{1}\left(P_{4}\right) ;\{x, y\} \in \Psi^{[2]}(4) ; w \in \Psi(4)\right]
$$

такими, что соответствуюшая прямая $(f, g)$ удовлетворяет ровно трем (соответственно, бесконечно многим) элементарным условиям. Прообразы этих двух множеств при очевидной проекции $\widetilde{\rho} \Sigma \rightarrow \widetilde{\lambda}(4)$ обозначим через $\{3\}$ и $\{\infty\}$ и вычислим по отдельности их замкнутые гомологии.

4.3.1. Гомологии $\{3\}$. Каждой прямой $(f, g)$, удовлетворяющей ровно трем элементарным условиям, соответствует шесть треугольников (11) в $\widetilde{\lambda}(4)$, объединение которых является открытым треугольником с барицентром в точке $(f, g)$ : граница этого треугольника лежит в $F_{1.5}$ (а вершины даже в $F_{1}$ ).

ПРЕДЛОЖЕНИЕ 7. Множество прямых $(f, g) \in G_{1}\left(P_{4}\right)$, все элементы которых удовлетворяют ровно трем общим әлементарным условиям, гомеоморфно прямому произведению прямой $\mathbb{R}^{1}$, интервала $(-\infty, 0)$ и плоского множества, ограниченного кривой, изображенной на рис. 1а) (әде пунктирная линия обозначает часть границы, не принадлехсащую множеству). В частности, замкнутые гомологии этого множсества тривиальны.

СледСТвиЕ. $\bar{H}_{*}(\{3\})=0$. 

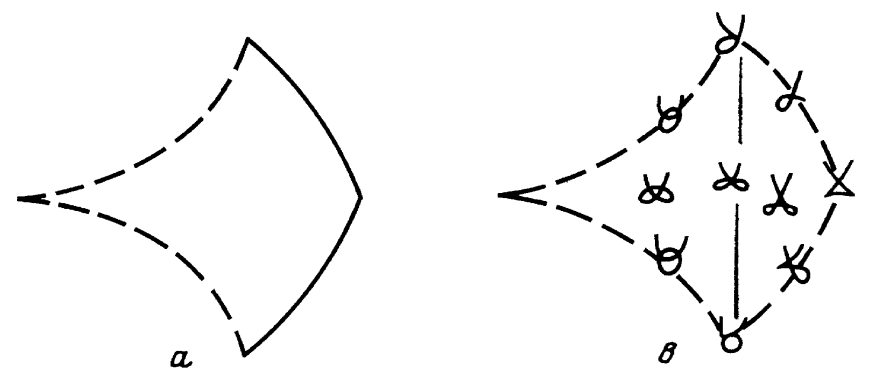

Рис. 1. Множество полиномов $f$, кривые отношений которых пересекают $r(g)$ в трех точках

ДокАЗАТЕЛьство СЛЕДствИЯ. Действительно, $\{3\}$ есть пространство расслоения над рассмотренным в предыдушем предложении подмножеством в $G_{1}\left(P_{4}\right)$, слой которого является прямым произведением $n$-мерного аффинного пространства (n-й степени тавтологического расслоения) и открытого треугольника.

ДоКАЗАТЕЛЬСТво ПРЕДЛОЖЕНИЯ 7 . По теореме Безу, если пара $(f, g)$ удовлетворяет ровно трем условиям, то $\operatorname{deg} g=3$, т.е. $g=t^{3}+\alpha t^{2}+\beta t$, a $r(g)$ имеет вид

$$
\left(t^{2}+t s+s^{2}\right)+\alpha(t+s)+\beta=0
$$

Группа сдвигов аргумента $t$ свободно действует на исследуемом множестве: это действие определяет первый сомножитель $\mathbb{R}^{1}$ в предложении 7. Следовательно, достаточно рассматривать лишь пространство пар $(f, g)$ с коэффициентом $\alpha$ в $g$ равным нулю: это пространство может быть естественно отождествлено с множеством орбит этого действия. Коэффициент $\beta$ в (12), очевидно, обязан быть отрицательным; его значения определяет сомножитель $(-\infty, 0)$ в предложении 7. Нам остается доказать лишь следуюшую лемму.

ЛЕмма 7. Для любого отрицательного значения $\beta$ множество полиномов $f=t^{4}+a t^{2}+b t$ таких, что соответствующая кривая отношений

$$
r(f) \equiv\left\{(t, s) \mid\left(t^{3}+t^{2} s+t s^{2}+s^{3}\right)+a(t+s)+b=0\right\}
$$

пересекает кривую

$$
r(g) \equiv\left\{(t, s) \mid\left(t^{2}+t s+s^{2}\right)+\beta=0\right\}
$$

ровно в трех точках, гомеоморфно множеству, указанному на рис. 1а). Объединение таких множеств по всем значениям $\beta$ определяет локально тривиальное расслоение над множеством $(-\infty, 0)$ таких значений.

ДокАЗАтЕльство. Группа $\mathbb{R}_{+}$действует на множестве канонических пар $(f, g)$ с $\operatorname{deg} g=3$ по правилу

$$
T_{\lambda}(f(t), g(t))=\left(\lambda^{-4} f(\lambda t), \lambda^{-3} g(\lambda t)\right)
$$


это действие сводит доказательство первого утверждения леммы 7 к случаю $\beta=-1$ и доказывает утверждение о локальной тривиальности.

Пара уравнений (13), (14) с $\beta=-1$, переписанная в симметрических функциях $p=t+s$ и $q=t s$, преврашается в систему

$$
\left\{\begin{array}{l}
p\left(p^{2}-2 q\right)+a p+b=0 \\
p^{2}-q=1 \\
p^{2}-4 q \geqslant 0
\end{array}\right.
$$

где неравенство отвечает за вешественность решений. Исключая $q$, получаем систему

$$
\begin{aligned}
& p^{3}-p(a+2)-b=0, \\
& p^{2} \leqslant 4 / 3 .
\end{aligned}
$$

Нас интересуют значения $a$ и $b$, при которых эта система имеет ровно три вешественных решения. Положим $A=a+2$, тогда множество пар $(A, b)$, для которых уравнение (17) имеет три вещественных корня, это область, ограниченная полукубической параболой

$$
27 b^{2} \leqslant 4 A^{3}
$$

см. рис. 1а). Эта область заметается параметрическими кривыми $\left\{A=\tau^{2}\right.$, $\left.b=C \cdot \tau^{3}\right\}, \tau \in(0,+\infty)$ с $C<\sqrt{4 / 27}$. Для любой такой кривой квадраты всех корней (17) монотонно возрастают вдоль нее, следовательно, эта кривая покидает множество, удовлетворяющее условию (18), раз и навсегда. Граница этого множества внутри области (19) состоит из двух компонент, соответствующих знаку корня (17), покидающего это множество в соответствующей точке: эти две компоненты встречаются на прямой $\{b=0\}$, на которой абсолютные значения двух корней совпадают.

Эта часть границы множества значений $\{a, b\}$, выделенных условиями $(17),(18)$, принадлежит области, изучаемой в лемме 7: действительно, ее точки соответствуют парам полиномов $(f, g)$ имеющих одну (или две в точке излома) общие особые точки и две (соответственно, одну) точку самопересечения. (Топологический вид плоских кривых, заданных отображениями $(f, g): \mathbb{R}^{1} \rightarrow \mathbb{R}^{2}$ для различных значений $A$ и $b$, изображен на рис. $1 \mathrm{~b})$.) $\mathrm{C}$ другой стороны, часть границы, на которой неравенство (19) становится равенством, не принадлежит интересуюшему нас множеству: в его точках две точки самопересечения сливаются и становятся одной точкой самокасания. Это доказывает лемму 7.

СЛЕДСТВИЕ. Если все полиномы аффинной прямой $(f, g) \subset P_{4}$ удовлетворяют ровно трем общим әлементарным условиям, то для любого $\lambda \in \mathbb{R}$

1) полином $f+\lambda g$ этой прямой имеет три вещественных критических точки (по меньшей мере две из которых геометрически различнь), любой отрезок в $\mathbb{R}^{1}$, соединяющий две критические точки такого полинома $f+\lambda g$, содержит критическую точку $g$;

2) взаимное расположсение кривых отношений $r(f+\lambda g)$ u $r(g)$ топологически устроено как показано на рисунках $2 \mathrm{a}$ ), b) или на картинках, симметричньх им при отражсении относительно вертикальной линии $\{t+s=\mathrm{const}\}$, 
или является их вырождением, соответствующим либо симметрическим полиномам $f+\lambda g$ (см. рис. 2 с), d)), либо совпадению некоторых критических точек $f$ с критическими точками $g$ (в последнем случае $r(f+\lambda g)$ u $r(g)$ пересекаются в своих концевых точках).

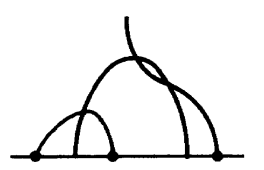

$\boldsymbol{a}$

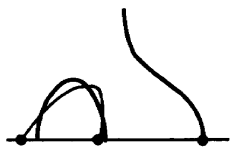

$\boldsymbol{B}$

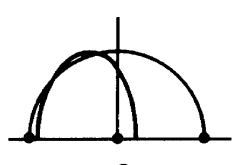

c

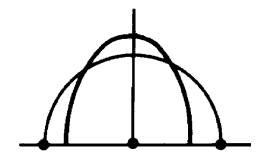

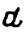

Рис. 2. Кривые отношений с тремя точками пересечения

ДокАЗАТЕЛьство. При $\lambda=0$ и $g$ вида $t^{3}+\beta t$ утверждение 1 вытекает из связности множества, исследуемого в лемме 7 . Для произвольных $\lambda$ то же самое следует из теоремы Ролля, поскольку в любом корне $g^{\prime}$ знаки всех полиномов вида $(f+\lambda g)^{\prime}$ с данным $f$ совпадают. Наконец, случай произвольных $\lambda$ и $g$ сводится к предыдущему подходящим сдвигом аргументов.

Второе утверждение вытекает непосредственно из первого.

4.3.2. Замкнутые гомологии $\{\infty\}$. Рассмотрим произвольное многообразие $S$ и вложим его общим образом в пространство $\mathbb{R}^{N}$ достаточно большой размерности, так что два различных отрезка, соединяющие образы точек $S$, могут пересекаться только в своих концевых точках. Обозначим объединение таких отрезков через $S^{* 2}$. Пространство $S$ канонически вложено в него.

Лемма 8. Если $S$ компактно, то $S^{* 2}$ компактно. Гомотопический тип пары $\left(S^{* 2}, S\right)$ определяется гомотопическим типом $S$.

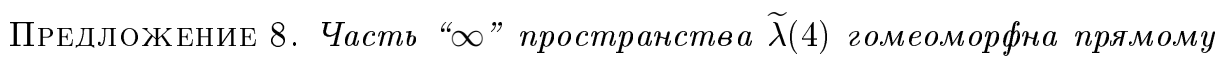
произведению прямой $\mathbb{R}^{1}$ и открытого конуса над пространством $S^{* 2}$, где $S$ - это отрезок $[0,+\infty]$.

ДокАЗАТЕЛьство. Если множество $r(f) \cap r(g)$ бесконечно, то по лемме 3 $\operatorname{deg} g=2$, и это множество является замкнутым отрезком в $\Psi(4)$, заданным уравнением $t+s=2 t_{0}$ для некоторого $t_{0}$; этот коэффициент $t_{0}$ определяет первый сомножитель $\mathbb{R}^{1}$ в предложении 8. Структура конуса вытекает из конструкции разрешения.

СледСтвие. $\bar{H}_{*}(\{\infty\})=0$.

ДоказАТЕльство. Множество $\{\infty\}$ является пространством $n$-мерного аф-

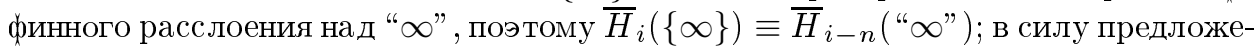
ния 8 последняя группа совпадает с группой замкнутых гомологий прямого произведения прямой линии и полуинтервала.

Теорема 2 полностью доказана.

4.4. Столбец $E_{1}^{-3, *}$. В этом разделе мы доказьваем следуюшую теорему. 
TEOpema $3 . E_{1}^{-3, *} \equiv 0$.

На этом шаге мы рассматриваем все подпространства коразмерности три в $P_{4}$, т.е. отдельные точки. Для любой такой точки $f$ мы рассматриваем полный прообраз в $F_{2}$ отображения $(f, \ldots, f) \in \mathscr{K}_{4}^{n}$ при очевидной проекции $\pi: \rho \Sigma \rightarrow \Sigma$ и берем открытьй конус над этим прообразом. Такие конусы, соответствующие всем $f \in P_{4}$, заметают член $F_{3} \backslash F_{2}$ разрешения $\rho \Sigma$; аналогичный член в $\tilde{\rho} \Sigma$ заметается такими же конусами, соответствующими тем $f$, которые принадлежат подмножеству $C(4,0) \subset P_{4}$.

Используя, как ранее, свободное действие групшы сдвигов пространства аргументов, мы можем рассматривать только полиномы $f$ вида $t^{4}+a t^{2}+b t$, т.е. с нулевым вице-старшим коэффициентом: все пространство $F_{3} \backslash F_{2}$ является прямым произведением прямой $\mathbb{R}^{1}$ и подпространства, заметенного конусами, соответствующими таким $f$.

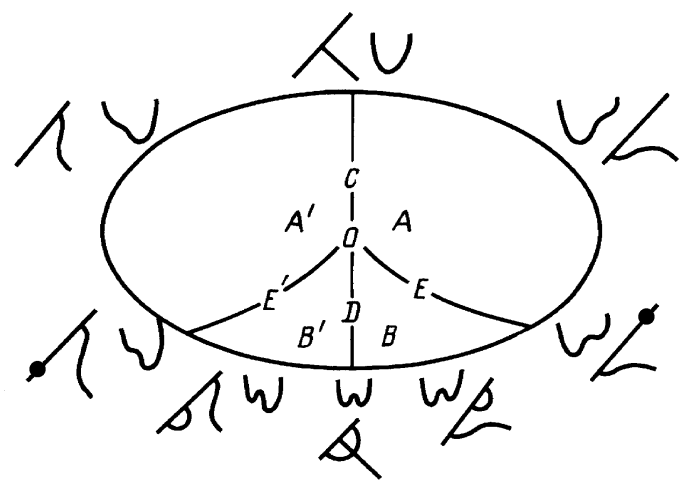

Рис. 3. Классификация полиномов четвертой степени

Все такие полиномы образуют двумерную плоскость, которая естественно разбивается на 9 открытых клеток, см. рис. 3. Для каждой из этих клеток мы приводим на этом рисунке топологический тип соответствуюших полиномов и кривых отношений. Клетки $A$ и $A^{\prime}$ (соответственно, $B$ и $B^{\prime}$ ) содержат строго морсовские полиномы с одной (соответственно, тремя) вещественными критическими точками. Они разделяются полукубической параболой $\left\{27 b^{2}+8 a^{3}=0\right\} \equiv E \cup E^{\prime} \cup O$ и прямой $\{b=0\} \equiv D \cup C \cup O$, состоящей из четных полиномов.

Для любой из девяти клеток мы рассматриваем объединение отображений $(f, \ldots, f) \in \Sigma \subset \mathscr{K}_{4}^{n}$, где $f$ пробегает эту клетку, и берем полный прообраз в $F_{3} \backslash F_{2}$ этого объединения относительно проекции

$$
\widetilde{\pi}: \widetilde{\rho} \Sigma \rightarrow \Sigma
$$

Обозначим этот прообраз через $\{X\}$, где $X$ - обозначение клетки, $X=A, A^{\prime}, \ldots$

4.4.1. Клетки $C$ и $O$. Все точки $f$ луча $\{b=0, a \geqslant 0\}$ не принадлежат $C(4,0)$ : действительно, для таких $f$ отрезок $r(f)$ совпадает с множеством пересечения кривых отношений всех полиномов, принадлежащих целой прямой $\{b=0\}$. Следовательно, в этом случае $\widetilde{\pi}^{-1}(f, \ldots, f) \cap\left(F_{3} \backslash F_{2}\right)=\varnothing$. 
ПРЕДЛОЖЕНИЕ 9. ДЛя любой клетки $X$, отличной от $C$ и $О$, множество $\{X\}$ является пространством локально тривиального расслоения над нашей клеткой, слой которого над $f$ есть открытый конус над множеством $\tilde{\pi}^{-1}(f, \ldots, f) \cap F_{2}$.

Для каждой клетки это немедленно вытекает из следуюшего ниже явного описания этого множества.

\subsection{2. Клетки $A$ и $A^{\prime}$.}

ЛЕмма 9. Если полином $f=t^{4}+a t^{2}+b t$ имеет только одну вещественную критическую точку, то $\tilde{\pi}^{-1}(f, \ldots, f) \cap\left(F_{2} \backslash F_{1.5}\right)=\varnothing$.

Доказательство вытекает немедленно из следствия леммы 7.

СлеДСТВИЕ. Для любого $f$ из клеток $A$ и $A^{\prime}$, множество $\tilde{\pi}^{-1}(f, \ldots, f) \cap$ $\left(F_{3} \backslash F_{2}\right)$ гомеоморфно открытому конусу над множсеством $S^{* 2}$ (см. п. 4.3.2), где $S$ - это кривая отношений $f$ (в частности, $S$ гомеоморфно отрезку).

СлЕДСТВИЕ. Группь замкнутых гомологий части $\{A\}$ или $\left\{A^{\prime}\right\}$ множества $F_{3} \backslash F_{2}$ тривиальны во всех размерностях.

Доказательство немедленно вытекает из формулы Кюннета и леммы 8.

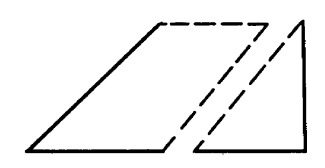

$a$

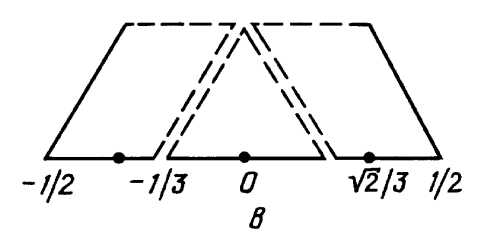

Рис. 4. Множество прямых, удовлетворяющих ровно трем элементарньм условиям и проходящих через $f, f \in B$ или $D$

\subsection{3. Клетки $B$ и $B^{\prime}$.}

ПРЕДЛОЖЕНИЕ 10. Для любого $f$ из клеток $B$ и $B^{\prime}$ множество аффинных прямых $(f, g) \subset P_{4}$, содержащих $f$, и таких, что все полиномы прямой удовлетворяют ровно трем общим әлементарным условиям, состоит из двух компонент и гомеоморфно множеству, ограниченному многоугольниками на рис. 4а) (әде пунктирная линия обозначает часть границь, не принадлежсащую множеству).

Доказательство этого предложения и следующих предложений 11, 12 см. в $\S 5$.

СлЕДСТВИЕ. Группьь $\bar{H}_{*}(\{B\}) u \bar{H}_{*}\left(\left\{B^{\prime}\right\}\right)$ тривиальны.

ДокАЗАТЕЛЬСТво. В силу предложения 9 и формулы Кюннета достаточно доказать, что для любого $f$ из нашей клетки группа замкнутых гомологий любого множества $\tilde{\pi}^{-1}(f, \ldots, f) \cap F_{2}$ тривиальна. Эти гомологии совпадают с гомологиями $\widetilde{\pi}^{-1}(f, \ldots, f) \cap F_{1.5}$ : действительно, в силу предложения 10 аналогичные гомологии $\widetilde{\pi}^{-1}(f, \ldots, f) \cap\left(F_{2} \backslash F_{1.5}\right)$ тривиальны. Из определения $F_{1.5}$ следует, что последняя группа совпадает с аналогичной группой множества $S^{* 2}$, где $S$ - кривая $r(f)$, т.е. несвязное объединение двух отрезков. Отсюда вытекает наше следствие. 


\subsection{4. Клетки $E$ и $E^{\prime}$.}

ПРЕДЛОЖЕНИЕ 11. Для любого $f$ из клеток $E$ и $E^{\prime}$, множество аффинных прямых $(f, g)$ в $P_{4}$, проходящих через $f$, и таких, что все полиномы прямой удовлетворяют ровно трем общим әлементарным условиям, гомеоморфно полуинтервалу.

СлЕДСТВИЕ. Группьь $\bar{H}_{*}(\{E\}) u \bar{H}_{*}\left(\left\{E^{\prime}\right\}\right)$ тривиальны.

ДоКАЗАТЕЛЬство - то же самое, что у следствия предложения 10 (в нашем случае кривая отношений $f$ является несвязным объединением отрезка и точки).

\subsection{5. Клетка $D$.}

ПРЕДЛОЖЕНИЕ 12. Для любого $f$ из клетки $D$ множсество аффинных прямых $(f, g)$ в $P_{4}$, проходящих через $f$, и таких, что все полиномы прямой удовлетворяют ровно трем общим әлементарным условиям, состоит из трех связных компонент и гомеоморфно ограниченному мнохсеству на рис. 4b) (әде пунктирный отрезок обозначает часть гранищы, не принадлежсащую множеству, а двойные пунктирные отрезки внутри фигуры должни быть выброшены из нее).

СлеДСТВИЕ. Группа $\bar{H}_{*}(\{D\})$ тривиальна.

ДоКАЗАТЕЛЬСТво - то же самое, что у следствия из предложения 10 (при $f \in D$ кривая $r(f)$ гомеоморфна замкнутому кресту и следовательно $r(f)^{* 2} \sim\{$ точка $\left.\}\right)$.

Теорема 3 вытекает немедленно из следствий леммы 9 и предложений 10, 11 и 12; она завершает доказательство теоремы 1.

\section{§5. Доказательство предложений 10, 11 и 12}

Пусть $f=t^{4}+a t^{2}+b t$ - точка клетки $B$ или $B^{\prime}$. Обозначим через $x_{1}<x_{2}<x_{3}$ три корня полинома $f^{\prime} \equiv 4 t^{3}+2 a t+b$; очевидно $x_{1}+x_{2}+x_{3}=0$. Достаточно рассмотреть только одну из клеток $B, B^{\prime}$, скажем $B$. Тогда $x_{1}$ отрицательно, $x_{2}$ и $x_{3}$ положительны, и топологически кривая $r(f)$ устроена так, как показано на рис. $2 \mathrm{a})$, так что $x_{2}$ и $x_{3}$ соединены ее "конечной" ветвью. Прямая $\{t+s=0\}$ является асимптотой для $r(f)$, в частности, она не пересекает ее в конечных точках и разделяет две ее ветви.
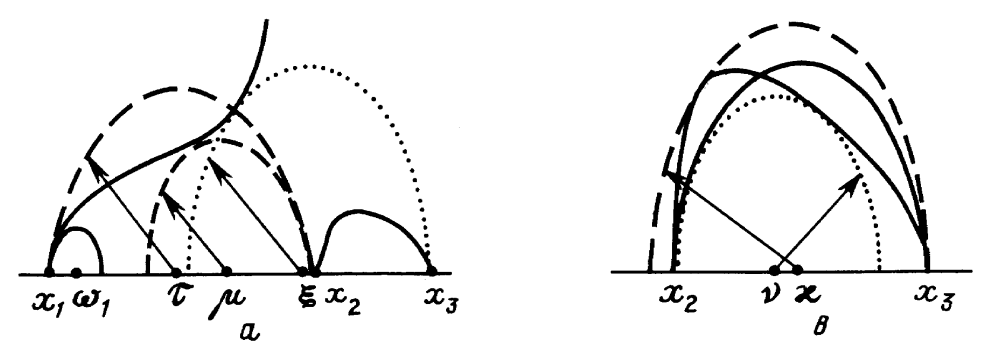

Рис. 5. Кривая отношений полинома $f$ типа $B$ и критические положения $r(g)$ 
В силу следствия из леммы 7 , если $\#(r(g) \cap r(f))=3$, то топологически расположение этих кривых устроено как на рис. 2a) или $2 \mathrm{~b}$ ) (или на вырождении этой картинки, соответствуюшем совпадению одной или нескольких концевых точек $r(g)$ с таковыми для $r(f))$.

Обозначение. Для любого $f \in B$ обозначим через $\varnothing \equiv \varnothing(f)$ (соответственно, через $\diamond)$ множество таких полиномов

$$
g=t^{3}+\alpha t^{2}+\beta t
$$

что множество $r(f) \cap r(g)$ состоит из трех точек, две из которых принадлежат "бесконечной" ветви $r(f)$ (соответственно, все три точки принадлежат "конечной" ветви).

Tеорема 4. Для любого $f \in B$ множество $\varnothing$ (соответственно, $\diamond)$ гомеоморфно плоскому множеству, ограниченному правой (соответственно, левой) компонентой на рис. 4а).

Доказательство этой теоремы занимает следующие пункты 5.1-5.3; предложение 10 является ее прямьм следствием.

\section{1. Семейства $L^{\nabla}$ и фокальные точки.}

Обозначение. Для любого $\nabla \in \mathbb{R}^{1}$ обозначим через $L^{\nabla}$ прямую в $P_{3}$, состоящую из полиномов (21) с $\alpha=-3 \nabla$, или, что то же самое, таких, что центр $r(g)-$ это точка $(\nabla, \nabla)$.

Кривые $r(g), g \in L^{\nabla}$, являются линиями уровня функции

$$
F_{\nabla} \equiv\left(t^{2}+t s+s^{2}\right)-3 \nabla(t+s)
$$

Лемма 10. Ограничение любой квадратичной формы в $\mathbb{R}^{2}$ на кубическую кривую имеет не более 9 изолированных критических точек.

Это следует немедленно из теоремы Безу.

СледствИЕ. Ограничение любой функции $F_{\nabla}$ на кривую $r(f) \subset \Psi(4), f \in B$, имеет не более трех критических точек строго внутри этой кривой.

ДокАЗАТЕльство. Кривая (13) сохраняется инволюцией $\{t \leftrightarrow s\}$, а три ее неподвижные точки $\left(x_{i}, x_{i}\right)$ являются критическими для любой $F_{\nabla}$.

Лемма 11. Существуют значения $\omega_{1}>x_{1}, \omega_{2}>x_{2} u \omega_{3}<x_{3}$ такие, что если $\nabla$ лежит с той же стороны от $\omega_{i}$, что и $x_{i}$ (соответственно, $\omega_{i}$ разделяет $\nabla$ и $\left.x_{i}\right)$, то ограничение функиии $F_{\nabla}$ на $r(f)$ имеет в точке $x_{i}$ сильный (квадратичный) локальный минимум (соответственно, максимум). А именно,

$$
\omega_{1}=\frac{6 x_{1}^{2}-a}{12 x_{1}}, \quad \omega_{2}=\frac{6 x_{2}^{2}-a}{12 x_{2}}, \quad \omega_{3}=\frac{6 x_{3}^{2}-a}{12 x_{3}} .
$$


ДоказАТЕЛьство. $\left(\omega_{i}, \omega_{i}\right)$ суть фокальные точки пар $\left(r(f),\left(x_{i}, x_{i}\right)\right)$ в смысле [7] относительно евклидовой метрики $t^{2}+t s+s^{2}$. Конкретные значения (23) находятся прямьми вычислениями.

5.2. Структура множества $\oslash$. Для любого $\nabla \in \mathbb{R}^{1}$ мы рассмотрим перестройки кривых $r(g)$ при $g$, пробегаюшем прямую $L^{\nabla}$. При $\beta=3 \nabla^{2}$ такая кривая состоит из единственной точки $(\nabla, \nabla)$, но когда параметр $\beta$ уменшшается, эта кривая раздувается вокруг того же центра. Ее расположение относительно $r(f)$ может претерпеть следуюшие четыре существенные для нас перестройки:

$M_{i}(i=1,2,3):$ точка пересечения $r(g)$ с диагональю $\{t=s\}$ проходит через $\left(x_{i}, x_{i}\right)$ - точку пересечения $r(f)$ с диагональю;

$M_{4}$ (возможная): кривая $r(g)$ касается "бесконечной" (т.е. лежащей в области $\{t+s<0\}$ ) ветви $r(f)$ в некоторой ее внутренней точке (и пересекает эту ветвь в последуюшие моменты, соответствующие бо́льшим эллипсам или меньшим значениям $\beta$ ).

Обозначим значения $\beta$, при которых происходят эти перестройки, через $\beta_{1}, \ldots, \beta_{4}$, соответственно; они являются функциями от $\nabla$ (или от $\alpha \equiv-3 \nabla$ ) и от коэффициентов полинома $f$. Полином $g$ вида $(21)$ принадлежит $\odot$ тогда и только тогда, когда выполнены следующие условия:

а) перестройка $M_{4}$ в семействе $L^{\nabla}$ действительно происходит в некоторый момент $\beta_{4}(\alpha)>\beta$;

b) $\beta \geqslant \beta_{1}(\alpha) ; \beta_{2}(\alpha) \geqslant \beta ; \beta \geqslant \beta_{3}(\alpha)$.

Для данного $\alpha$ множество значений $\beta$, для которых полином (21) удовлетворяет условиям a), b), может быть пусто, или состоять из единственной точки (если $\beta_{4}(\alpha)>\beta_{2}(\alpha)=\beta_{1}(\alpha)>\beta_{3}(\alpha)$ ), быть замкнутым отрезком (если $\left.\beta_{4}(\alpha)>\beta_{2}(\alpha)>\max \left(\beta_{1}(\alpha), \beta_{3}(\alpha)\right)\right)$ или полуинтервалом (если $\beta_{2}(\alpha)>\beta_{4}(\alpha)>$ $\left.\max \left(\beta_{1}(\alpha), \beta_{3}(\alpha)\right)\right)$.

Сейчас мы найдем значения $\alpha$, при которых эти условия выполнены.

Лемма 12. Перестройка $M_{4}$ происходит в некоторый момент $\beta_{4}(\alpha)>$ $\beta_{1}(\alpha)$, если и только если $\nabla>\omega_{1}$.

5.2.1. Точки бифуркаций. Сейчас для любого фиксированного $f \in B$ мы определим три важные точки на прямой $\mathbb{R}^{1}$ значений $\nabla$, см. рис. $\left.5 \mathrm{a}\right)$.

Положим $\tau=\left(x_{1}+x_{2}\right) / 2$, так что $(\tau, \tau)$ является центром эллипса вида $(12)$, пересекающего диагональ в точках $\left\{x_{1}, x_{1}\right\}$ и $\left\{x_{2}, x_{2}\right\}$.

Семейство эллипсов (12), пересекающих диагональ в двух точках, бо́льшая из которых (т.е. ближайшая к $(+\infty,+\infty))$ есть $\left(x_{3}, x_{3}\right)$, является однопараметрической кривой в плоскости $\{\alpha, \beta\}$; все эллипсы этого семейства лежат один внутри другого. Рассмотрим наименьший эллипс этого семейства, пересекающий "бесконечную" ветвь $r(f)$, и обозначим через $\xi$ соответствующее значение $-\alpha / 3$, так что $(\xi, \xi)$ есть центр этого эллипса. Наконец, точка $\mu$ определяется аналогичным образом при помощи семейства эллипсов $(12)$, содержащих точку $\left(x_{2}, x_{2}\right)$.

ЛЕмма 13. $\tau>\omega_{1}$.

ДокАЗАТЕЛЬСтво. По лемме 11 (и поскольку $x_{1}<0$ ) это утверждение эквивалентно неравенству $6 x_{1} x_{2}+a<0$. Но при $f \in B$ и $a$, и $x_{1} x_{2}$ отрицательны. 
СлЕДСТвИЕ 1. $\mu>\tau$.

ДокАЗАТЕЛЬСТво. По определению $\mu \geqslant \tau$. Но если $\mu=\tau$, то эллипс $r(g)$, проходящий через $\left(x_{2}, x_{2}\right)$ и с центром в $(\mu, \mu)$, пересекает диагональ также и в точке $\left(x_{1}, x_{1}\right)$. В силу леммы 13 он ограничивает континуум точек "бесконечной" ветви, что противоречит определению $\mu$.

СлеДСТвиЕ 2. $\xi>\mu$.

ДоКАЗАТЕЛЬСТво. Если $\xi \leqslant \mu$, то эллипс (12), проходящий через $\left(x_{2}, x_{2}\right)$ и с центром в $(\mu, \mu)$ (участвующий в определении точки $\mu$ ), лежит строго внутри эллипса, проходящего через $\left(x_{3}, x_{3}\right)$ и с центром в $(\xi, \xi)$. Поскольку первый эллипс имеет непустое пересечение с "бесконечной” ветвью $r(f)$, последний ограничивает континуум точек этой ветви, что противоречит определению $\xi$.

Окончательно, мы получаем, что наши точки упорядочены следуюшим образом: $\omega_{1}<\tau<\mu<\xi$. Прямая $L^{\nabla}$ пересекает область $\bigcirc$ по

- точке, если $\nabla=\tau$,

- отрезку, если $\nabla \in(\tau, \mu)$,

- полуинтервалу, если $\nabla \in[\mu, \xi)$,

- пустому множеству, если $\nabla \notin[\tau, \xi)$.

Отсюда вытекает утверждение теоремы 4 о множестве $\varnothing$.

ЗАмЕчАниЕ. В этом доказательстве мы рассматривали слои отображения $\odot \rightarrow \mathbb{R}^{1}$, переводящего $g$ в центр симметрии $g^{\prime}$. Используя другое отображение $\curlyvee \rightarrow \mathbb{R}^{1}$, переводящее $g$ в его наибольшую критическую точку, мы можем установить гомеоморфизм $\odot$ на прямое произведение отрезка и полуинтервала. Действительно, в силу следствия леммы 7 эта критическая точка должна принадлежать отрезку $\left[x_{2}, x_{3}\right]$, а в силу следствия 1 леммы 13 для любой точки $x$ этого отрезка слой нашего отображения над $x$ гомеоморфен полуинтервалу.

\section{3. Структура множества $\diamond$.}

Лемма 14. Если прямая $L^{\nabla}$ имеет непустое пересечение с множеством $\diamond, m o \nabla \leqslant\left(x_{2}+x_{3}\right) / 2$.

Действительно, это вытекает из следствия леммы 7.

Лемма 15. $\left(x_{2}+x_{3}\right) / 2<\omega_{2} u\left(x_{2}+x_{3}\right) / 2<\omega_{3}$.

ДокАЗАТЕЛЬСТво. В силу (23) (и поскольку $x_{3}>0<x_{2}$ ) оба эти неравенства эквивалентны неравенству $6 x_{2} x_{3}+a<0$. В силу формул Виета

$$
x_{1}+x_{2}+x_{3}=0, \quad x_{1} x_{2}+x_{1} x_{3}+x_{2} x_{3}=a / 2,
$$

оно эквивалентно неравенству $\left(x_{3}-x_{2}\right)^{2}>0$.

По теореме Ролля, если $r(g)$ имеет три точки пересечения с “конечной” ветвью $r(f), g \in L^{\nabla}$, то ограничение функции $F_{\nabla}$ на эту ветвь имеет по меншшей мере две критических точки в своей внутренней части.

ЛЕмма 16. Пусть $\nabla \leqslant\left(x_{2}+x_{3}\right) / 2$. Тогда ограничение функиии $F_{\nabla}$ имеет две критические точки внутри “конечной” области, если и только если $\nabla>\sqrt[3]{b} / 2$ 
ДокАЗАТЕЛЬСтво. Условие $d F_{\nabla} \| d f$ легко вычисляется и эквивалентно во внутренней части $\Psi(4)$ уравнению $\left(t^{2}+4 t s+s^{2}\right)+2 \alpha(t+s)-a=0$. Вместе с уравнением кривой $r(f)$ это дает следуюшую систему в симметрических функциях $p, q$ :

$$
\left\{\begin{array}{l}
p\left(p^{2}-2 q\right)+a p+b=0, \\
p^{2}+2 q+2 \alpha p-a=0, \\
p^{2}-4 q \geqslant 0
\end{array}\right.
$$

cp. (16). Два первых уравнения в ней дают следующее условие на $p$ :

$$
p^{3}+\alpha p^{2}+b / 2=0 \text {. }
$$

Если $\nabla=\left(x_{2}+x_{3}\right) / 2$, то $F_{\nabla}$ имеет две критических точки внутри “конечной" компоненты (в силу леммы 15 и поскольку $F_{\nabla}$ принимает одинаковые значения в концах этой компоненты); при $\nabla$ близких к $-\infty F_{\nabla}$ очевидно не имеет таких критических точек. Когда $\nabla$ движется от $\left(x_{2}+x_{3}\right) / 2$ к $-\infty$, число критических точек может уменьшаться, лишь если дискриминант полинома (26) обрашается в ноль или если последнее неравенство в (25) становится равенством. Последнее невозможно: действительно, это соответствует ситуации когда $t=s$, т.е. некоторая внутренняя критическая точка $\left.F_{\nabla}\right|_{r(f)}$ подходит к концу компоненты, что противоречило бы лемме 15. А предыдушее дискриминантное условие, очевидно, эквивалентно условию $\nabla=\sqrt[3]{b} / 2$.

Лемма 17. $x_{2}<\sqrt[3]{b} / 2<\left(x_{2}+x_{3}\right) / 2$.

ДокаЗАТЕЛЬСтво. Оба эти неравенства вытекают из формул Виета (24) и $b / 4=-x_{1} x_{2} x_{3}$ и из того, что $x_{3}>x_{2}$ и $-x_{1}>2 x_{2}$. (Правое неравенство следует также из доказательства предыдушей леммы.)

Итак, для любой точки $\nabla \in\left(\sqrt[3]{b} / 2,\left(x_{2}+x_{3}\right) / 2\right]$ ограничение функции $F_{\nabla}$ на конечную ветвь имеет четыре критических точки: минимумы в $\left(x_{2}, x_{2}\right)$ и некоторой точке $m(\nabla)$ и максимумы в $\left(x_{3}, x_{3}\right)$ и $M(\nabla)$. Обозначим через $\beta^{1}(\nabla), \ldots, \beta^{4}(\nabla)$ взятые со знаком минус критические значения в этих точках, так что все эллипсы $r(g), g=t^{3}-3 \nabla t^{2}+\beta^{i}(\nabla) t$, имеют касание с конечной ветвью $r(f)$. Пересечение нашей прямой $L^{\nabla}$ c $\diamond$ состоит из полиномов $(21)$ с $\alpha=-3 \nabla$ и $\beta$, удовлетворяющим обоим условиям $\beta^{1}(\nabla) \geqslant \beta \geqslant \beta^{3}(\nabla)$ и $\beta^{2}(\nabla)>\beta>\beta^{4}(\nabla)$. Поэтому, для того чтобы исследовать множество $\diamond$, нам остается установить порядок точек $\beta^{i}(\nabla)$ для различных $\nabla \in\left(\sqrt[3]{b} / 2,\left(x_{2}+x_{3}\right) / 2\right]$.

Для любого такого $\nabla$ критические значения в обеих точках максимума, очевидно, больше значений в минимумах, поэтому $\beta^{1}(\nabla)$ и $\beta^{2}(\nabla)$ больше чем $\beta^{3}(\nabla)$ и $\beta^{4}(\nabla)$.

Лемма 18. Для любого $f \in$ В существует точка $\varkappa \in\left(\sqrt[3]{b} / 2,\left(x_{2}+x_{3}\right) / 2\right)$ такая, что $\beta^{3}(\varkappa)=\beta^{4}(\varkappa), \beta^{3}(\nabla)>\beta^{4}(\nabla)$ при $\nabla>\varkappa u \beta^{3}(\nabla)<\beta^{4}(\nabla)$ при $\nabla<\varkappa$.

ДокаЗАтЕЛЬСтво. Рассмотрим однопараметрическое семейство полиномов (21) таких, что одна из их критических точек совпадает с $x_{3}$, а вторая меншше или равна $x_{2}$. Это семейство пересекает любую прямую $L^{\nabla}, \nabla \leqslant\left(x_{2}+x_{3}\right) / 2$, ровно в 
одной точке, следовательно, $\nabla$ является естественной координатой на нем. Пусть $g_{0}$ - начальная точка семейства (принадлежащая $L^{\nabla}, \nabla=\left(x_{2}+x_{3}\right) / 2$ ), тогда эллипс $r\left(g_{0}\right)$ пересекает конечную ветвь $r(f)$ в трех точках, две из которых суть $\left(x_{2}, x_{2}\right)$ и $\left(x_{3}, x_{3}\right)$, см. рис. $\left.5 \mathrm{~b}\right)$. Когда $\nabla$ убывает, эллипсы $r(g)$ семейства монотонно расширяются, в частности, начиная с некоторого момента $\varkappa$ они содержат эту ветвь $r(f)$ внутри. В этот момент соответствующий эллипс касается этой ветви в двух точках (одна из которых $\left(x_{3}, x_{3}\right)$ ), и критические значения $-\beta^{3}(\varkappa),-\beta^{4}(\varkappa)$ соответствующей функции $F_{\varkappa}$ совпадают. По теореме Ролля $F_{\varkappa}$ имеет еще одну критическую точку во внутренности нашей ветви, следовательно, в силу леммы 16 $\varkappa>\sqrt[3]{b} / 2$. Очевидно, это значение $\varkappa$ является искомьм.

Лемма 19. Для любого $f \in B$ найдется точка $\nu \in\left(\sqrt[3]{b} / 2,\left(x_{2}+x_{3}\right) / 2\right)$ такая, ито $\beta^{1}(\nu)=\beta^{2}(\nu), \beta^{1}(\nabla)>\beta^{2}(\nabla)$, если $\nabla<\nu u \beta^{1}(\nabla)<\beta^{2}(\nabla)$, если $\nabla>\nu$.

ДокАЗАТЕЛЬство повторяет доказательство леммы 18: мы рассматриваем семейство полиномов $g$ с одной критической точкой равной $x_{2}$ и другой, меньшей или равной $x_{3}$ (так что начальная функция $g_{0}$ - та же самая, что в предыдущем доказательстве, см. рис. 5b)).

Топологическая структура множества $\diamond$ вытекает из лемм 14-19. А именно, пересечение прямой $L^{\nabla}$ с этим множеством является точкой при $\nabla=$ $\left(x_{2}+x_{3}\right) / 2$, отрезком при $\nabla \in\left(\max (\varkappa, \nu),\left(x_{2}+x_{3}\right) / 2\right)$, полуинтервалом при $\nabla \in(\min (\varkappa, \nu), \max (\varkappa, \nu)]$, интервалом при $\nabla \in(\sqrt[3]{b} / 2, \max (\varkappa, \nu)]$ и пусто при $\nabla \notin\left(\sqrt[3]{b} / 2,\left(x_{2}+x_{3}\right) / 2\right]$.

5.4. Доказательство предложения 11. Множество, исследуемое в предложении 11, есть предел множеств $\varnothing=\varnothing(f)$, когда $f \in B$ стремится к клетке $E$, а, следовательно, отрезок $\left[x_{2}, x_{3}\right]$ стягивается в точку. Его структура вытекает из следствия 1 леммы 13.

5.5. Доказательство предложения 12. Группа растяжений (15) эффективно действует на клетке $D$ и свободно действует на соответствующей части $\widetilde{\rho} \Sigma$. Поэтому мы можем рассмотреть единственный полином $f=t^{4}-2 t^{2}$, так что $r(f)$ состоит из отрезка

$$
t+s=0
$$

и полуокружности

$$
t^{2}+s^{2}=2
$$

ЛЕмма 20. Мнохсество таких значений $\nabla$, что для некоторого $g \in L^{\nabla}$ кривая $r(g)$ пересекает полуокружность (28) дважды, есть интервал $(-2 / 3,2 / 3)$.

Это предельньй вариант леммы 11 (точнее, ее части, касающейся точек $x_{1}$ и $x_{3}$ ) при $f \in B$, стремяшемся к нашему полиному $t^{4}-2 t^{2}$.

Если $\nabla \in(1 / 2,2 / 3] \cup[-2 / 3,-1 / 2)$, то расширяюшиеся эллипсы семейства $L^{\nabla}$ пройдут через конечную точку $(1,1)$ или $(-1,-1)$ полуокружности $(28)$ раньше 


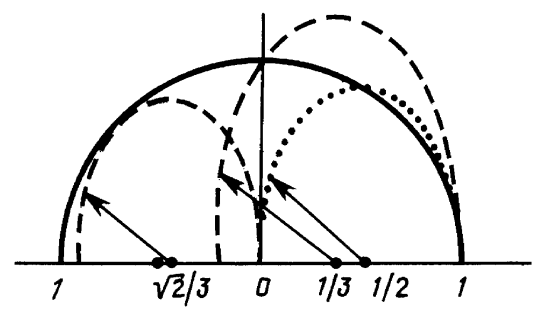

Рис. 6. Пересечения с симметричной кривой отношений

(при бо́льших значениях $\beta$ ), чем они наткнутся на компоненту (27), следовательно, такие семейства также не пересекают исследуемое множество.

При $\nabla= \pm 1 / 2$ пересечение семейства $L^{\nabla}$ с этим множеством состоит из единственного полинома $t^{3} \mp(3 / 2) t^{2}$, см. рис. 6 , а при $|\nabla|$ чуть меньших чем $1 / 2$, оно состоит из отрезка: действительно, перестройки взаиморасположений эллипсов $r(g)$ в таких семействах упорядочены следующим образом: сначала (при наибольшем значении $\beta$ ) они касаются полуокружности (28) в ее внутренней точке, затем впервые встречаются с отрезком (27) и после этого проходят через конщевую точку $(1,1)$ или $(-1,-1)$ полуокружности; отрезок семейства, соединяющий второе и третье происшествие - искомый.

Ситуация меняется, если первая и вторая перестройки происходят одновременно или даже меняют порядок: в этом случае граничная точка, в которой семейство входит в интересующее нас множество, не принадлежит этому множеству. Легко вычисляется, что это происходит при $|\nabla| \leqslant \sqrt{2} / 3$. Более того, любое семейство $L^{\nabla}$ имеет исключительную точку - эллипс $r(g)$, проходящий через особую точку $(-1,1)$ кривой $r(f)$, который пересекает $r(f)$ по меньшему числу точек, чем соседние эллипсы $r\left(g^{\prime}\right)$. Если $|\nabla| \leqslant 1 / 3, \nabla \neq 0$, то эта исключительная точка лежит внутри интервала интересующих нас точек семейства и поэтому должна быть выброшена из него; объединение таких точек изображается двойными пунктирными отрезками на рис. 4b).

\section{Список литературы}

1. Shastri A.R. Polynomial representations of knots // Preprint, January 1991.

2. Vassiliev V. A. Cohomology of knot spaces. Theory of Singularities and its Applications // Advances in Soviet Math. / ed. V. I. Arnold. V. 1. P. 23-69 (AMS, Providence, RI, 1990).

3. Vassiliev V.A. Complements of discriminants of smooth maps: topology and applications // Revised ed. Transl. of Math. Monogr. (AMS, Providence RI, 1994).

4. Арнольд В.И. О некоторых топологических инвариантах алгебраических функций // Труды ММО. 1970. Т. 21. С. 27-46.

5. Васильев В. А. Геометрическая реализация гомологий классических групп Ли и комплексы, $S$-двойственные к флаговьп многообразиям // Алгебра и Анализ. 1991. Т. 3. № 4 . C. $113-120$.

6. Vassiliev V.A. Invariants of ornaments. Singularities and Curves // Advances in Soviet Math. / ed. V.I. Arnold. V. 21. P. 225-262 (AMS, Providence, RI, 1994).

7. Милнор Джс. Теория Морса. М.: Мир, 1963.

Математический институт

им. В. А. Стеклова Р АН
Поступила в редакцию 27.07.1995 